\title{
Computer program for analysis of
}

parathyroid scintigraphy examinations: combination of dual-tracer (subtraction) and double phase single-tracer washout techniques

Małgorzata Iwona Kobylecka, Adam Bajera, Katarzyna Fronczewska-Wieniawska, Joanna Mączewska, Maria Teresa Płazińska, Leszek Królicki

Nuclear Medicine Department, Medical University of Warsaw

[Received 29 V 2015; Accepted 6 XI 2015]

\section{Abstract}

There is no controversy in the published literature that sensitivity and specificity of parathyroid scintigraphy is superior to other imaging techniques. However no uniform protocol has been established for scintigraphy. In order to analyze parathyroid scintigraphic images in the Department of Nuclear Medicine at the Medical University of Warsaw we have developed a program that allows qualitative and quantitative evaluation of recorded images and motion artifacts correction. This program offers a uniform procedure of analysis of parathyroid imaging results in diagnostic centers, accelerates the analysis of parathyroid tests performed with use of single radioactive tracer, that require the acquisition of consecutive images of the patient, without altering his body position between successive stages of registration. This program allows for automation of previously time consuming procedures and thus saves time and decreases a risk of operator's errors.

KEY words: parathyroids, MIBI, parathyroid scintigraphy, double phase MIBI scintigraphy

Nuclear Med Rev 2016; 19, 1: 58-62

Correspondence to: Małgorzata Iwona Kobylecka MD

Nuclear Medicine Department, Medical University of Warsaw

Bancha 1A, 02-097 Warsaw

Tel: 225991131 , fax: 225991170

E-mail: makob@onet.eu

\section{Background}

Scintigraphy is one of the basic imaging methods used in diagnosis of hyperfunctional parathyroid lesions. In the published literature, the authors are unanimous in the opinion that sensitivity and specificity of scintigraphy is superior to other imaging techniques; however, there is no uniform protocol how to perform the examination $[1,2]$

Imaging of hyperfunctioning parathyroid glands is mainly performed using both dual-tracer subtraction and double-phase single-tracer washout techniques. Neither of those techniques is optimal $[3,4]$.

In the Department of Nuclear Medicine at Medical University of Warsaw, parathyroid examinations using 99mTcMIBI have been performed since 1993. Based on our observations, we have noted that two-phase technique may lead to false negative and false positive results: some of the parathyroid adenomas are characterized by rapid washout of ${ }^{99 \mathrm{~m}} \mathrm{Tc}-\mathrm{MIBI}$ and some thyroid nodules may have enhanced and prolonged retention of the radiopharmaceutical.

This problem is particularly important in areas of endemic goiter [5]. For these reasons, in year 2000 we have decided to develop and modify our imaging technique and add the subtraction technique to initially performed two-phase protocol. Taking into consideration reduction of time of the study as well as of absorbed dose, scintigraphy is a one day examination and we use the protocol which combines both approaches, called combined protocol.

In order to analyze data, in our Nuclear Medicine Department we have developed our own examination protocol, as well as a program that allows qualitative and quantitative analysis of the images.

\section{Data registration protocol}

Subtraction and two-phase examinations were performed using combined protocol, with the initial part of the study being equivalent to subtraction examination and images obtained 20 min after administration of 99m Tc-MIBI regarded as images of "early" two-phase examination. Further stages of examination proceeded according 




Figure 1. Combined protocol scheme

to standard two-phase study protocol. Subtraction was performed by subtracting thyroid images obtained $10 \mathrm{~min}$ after administration of $60 \mathrm{MBq} \mathrm{Na}{ }^{99 m} \mathrm{TcO}_{4}$ from the image of "early" ${ }^{99 m} \mathrm{TC}-\mathrm{MIBI}$ standard two-phase parathyroid scintigraphy.

\section{Scan interpretation criteria}

Two-phase study scintigraphy was considered positive when focus of increased, abnormal accumulation of the radiopharmaceutical was found in early images and remained visible in late images with its intensity increasing in comparison with accumulation of the tracer in surrounding tissues (i.e. "extended tracer retention" in parathyroid) (Figure 1).

Subtraction study was considered positive whenever focus of tracer accumulation was found, regardless of its intensity.

Combined study was considered positive when focus of tracer accumulation met criteria of positive two-phase and/or subtraction study.

Introduction of subtraction technique using 99mTc/99m Tc-MIBI became possible only after planning and writing own correction program that would analyze obtained images. Such program enables effective elimination of motion artifacts which often cause false positive results.

\section{Computer program for analysis of parathyroid scintigraphy examinations}

The program is written in the XPert CLIP language, running on an APEX XPert, allowing fusion of the two-phase and subtraction study protocols in one analysis.

Program algorithm takes into account following facts:

- The procedure of digital matrix subtraction and necessity of ROI copying that is applied in analysis, requires the use of same size matrix during following acquisitions. We have adopted matrix128 x $128(1.8 \mathrm{~mm} /$ pix).

- Local amount of $99 \mathrm{mTc}$ in the area of salivary glands and mucous membranes may be several times higher than in the thyroid gland area, therefore it is necessary to limit quantitative analysis to the thyroid region.

- During a total of 35 minutes of two first sets of images acquisition it is possible that patient changes his position, which leads to motion artifacts resulting from the subtraction procedure of digital matrices, therefore it is necessary to correct the effects of patient's movements.
- Quantitative biodistribution of theNa99mTcO4 radiopharmaceuti-

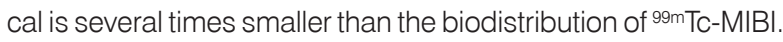
As far as imaging with aid of the scintigraphic camera is concerned, it includes only the distribution of the isotope. After the $1^{\text {st }}$ phase a digital matrix is a representation of ${ }^{99 \mathrm{~m} T \mathrm{C}}$ distribution, resulting from the biodistribution of $\mathrm{Na}^{99 \mathrm{~m}} \mathrm{TcO} 4$ radiopharmaceutical. However, after the $2^{\text {nd }}$ phase the digital matrix is a representation of the distribution of $99 \mathrm{mTc}$ both as a result of $\mathrm{Na}^{99 m} \mathrm{TcO} 4$ and ${ }^{99 m} \mathrm{TC}-\mathrm{MIBI}$ biodistribution. There is the ${ }^{99 m} \mathrm{Tc}$ biodistribution summation effect without distinguishing between the two radiopharmaceuticals biodistribution!

The aim of the proposed and clinically verified examination algorithm is to use the numeric criterion in the location of parathyroid and thyroid gland.

The program requires triple acquisition of thyroid and mediastinum areas images:

- first set of images - acquired 10 min after administration $\mathrm{Na}^{99 m \mathrm{TcO}} 4$;

- second set of images - 20 minutes after administration of

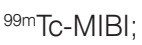

- third set of images - 120 min after administration of ${ }^{99 m} \mathrm{mC}-\mathrm{MIBI}$. Analysis of the examination using the proposed program consists of following steps (9-point smoothing has been used in all images):

1. Designation of region of quantitative analysis on the thyroid image (Figure 2).

2. Off-screen procedure of normalization of numerical values of all phases of the study to a common maximum value. The program was adapted to APEX system value MAX $=255$.

3. The result of the normalization of digital matrices to MAX value in order to select the area containing thyroid gland and delineation of thyroid isocontour on the 1st phase image (thyroid) (Figure 3).

4. Transfer of the isocontour to the 2nd phase image and indication of the area for normalization to the MAX value and quantitative analysis (Figure 4).

5. The correction of any error resulting from patient's movement by shifting the 2nd phase image. In order to facilitate the matching of images, a color scale is used that highlights the borders of the thyroid gland (Figure 5).

6. Image result of subtraction of the $2^{\text {nd }}$ phase from the $1^{\text {st }}$ phase digital matrices both normalized to the MAX value (Figure 6).

7. Arbitrary indication of small regions (ROI) on 2nd and 3rd images in the areas of the thyroid, parathyroid and background for quantitative analysis of ${ }^{99 \mathrm{~m}} \mathrm{Tc}-\mathrm{MIBI}$ retention. 


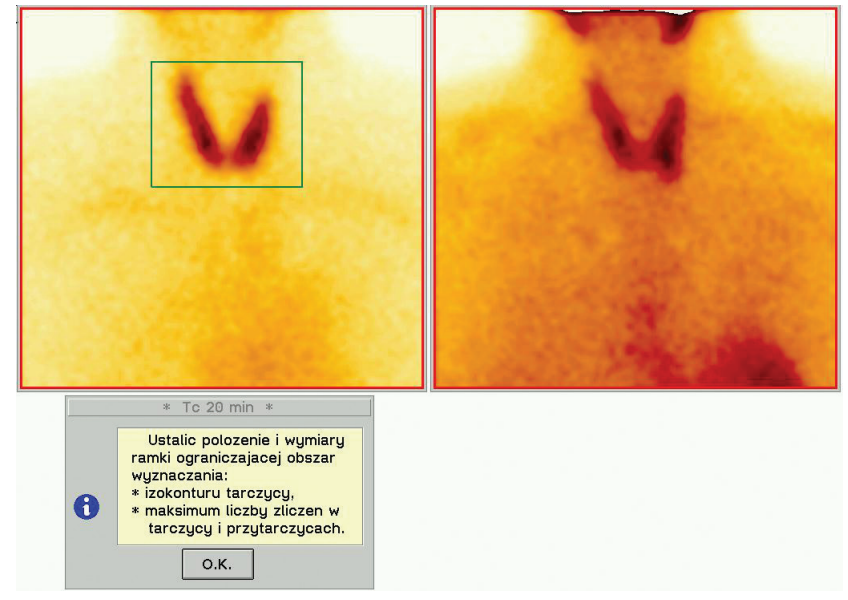

Figure 2. Scintigraphic scan of the neck. $\mathrm{Na}^{99 \mathrm{~m}} \mathrm{TcO}_{4}$ image on the left ${ }_{99 m} \mathrm{TC}-\mathrm{MIBI}$ image on the right. The grey window command: Adjust the location and dimension of the frame delineating: ROI (region of interest) for thyroid isocontour and of maximal count value in thyroid and parathyroids

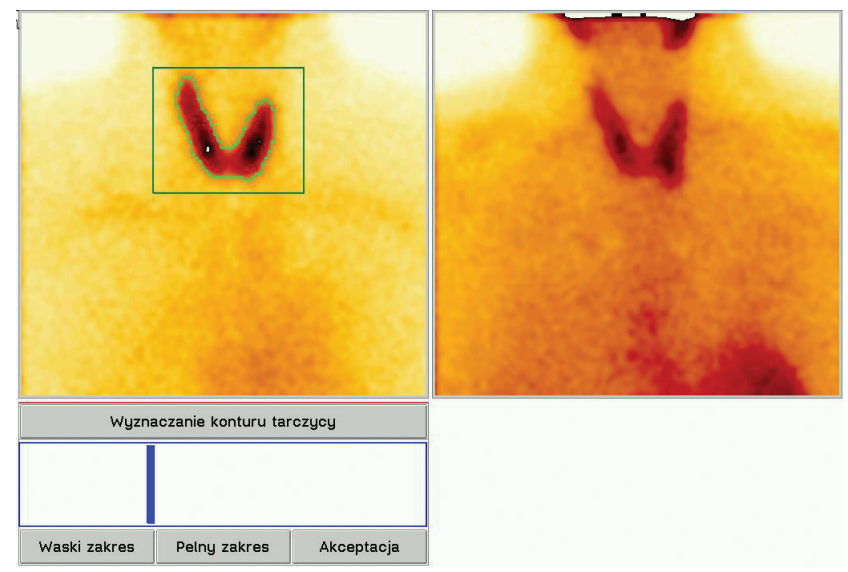

Figure 3. Thyroid isocontour drawn on the $\mathrm{Na}^{99 \mathrm{~m}} \mathrm{TcO}_{4}$ scan. The grey description: Wyznaczanie konturu tarczycy = Thyroid isocontour indication, Wąski zakres $=$ Narrow range, Pełny zakres $=$ Full range, Akceptacja $=$ Accept
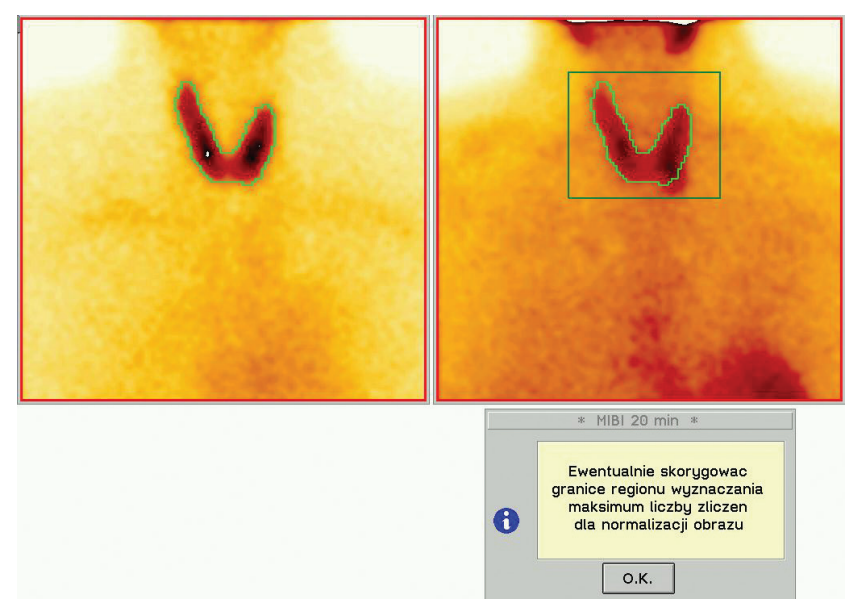

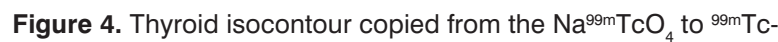
MIBI scan. scan. The grey window command: Eventually adjust the location and dimension of the frame delineating ROI for picture normalization



Figure 5. Adjustment of $99 \mathrm{~m} \mathrm{TC}-\mathrm{MIBI}$ to the transferred $\mathrm{Na}^{99 \mathrm{~m}} \mathrm{TcO}_{4}$ thyroid isocontour. The grey description: $\mathrm{W}$ lewo $=$ Left, $\mathrm{W}$ prawo $=$ Right, W dół $=$ Down, $W$ górę $=$ Up, Akcept. $=$ Accept
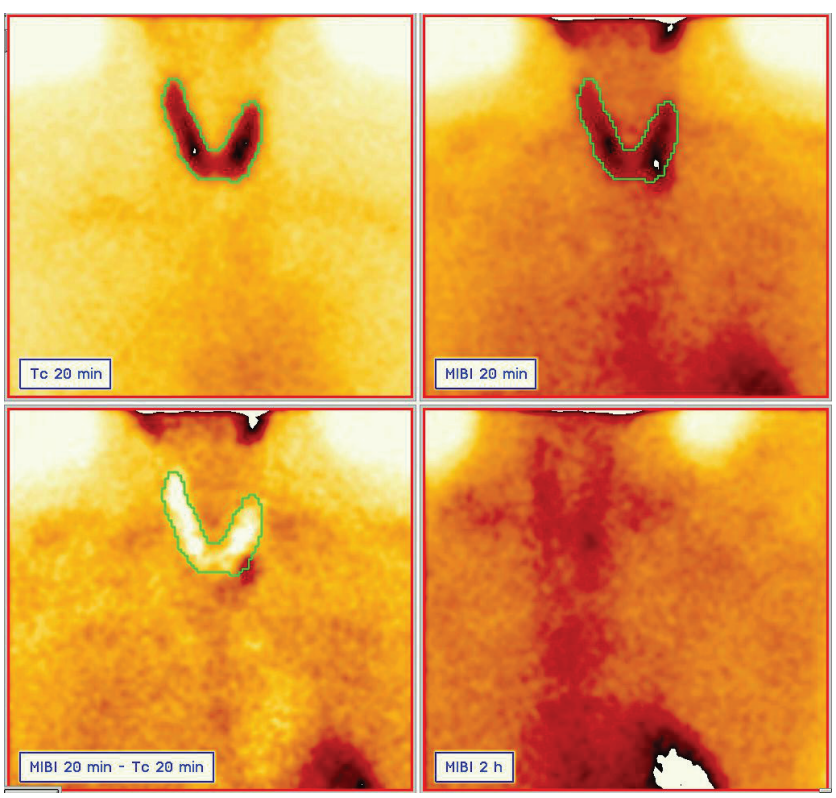

Figure 6. Full scintigraphic report after subtraction

The final report contains smoothed and normalized first and second images as well as subtraction image with superimposed thyroid gland isocontours. In addition, there is a third smoothed image. If the fourth step of the program is executed, predefined regions and a table of number of counts in these regions for the early and late images of 99m Tc-MIBI distribution are displayed (Figure 7).

Semiquantitative analysis offered by the program enables the comparison of 99m Tc-MIBI accumulation in parathyroid glands in relation to thyroid gland and background as well as relation of thyroid to background. The analysis is based on the designation of three identical small oval regions of interest (ROI-Region of Interest) in the area of: 


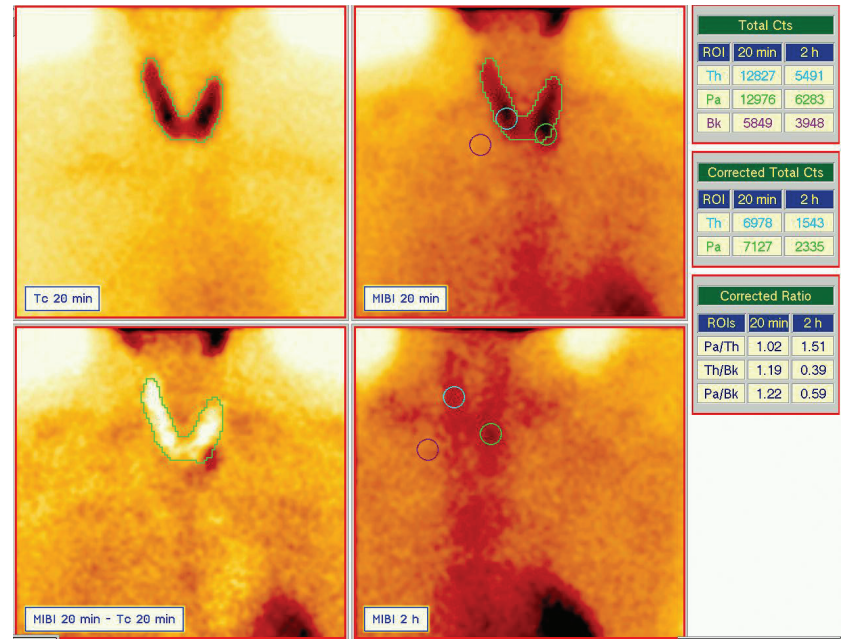

Figure 7. Full scintigraphic report with semiquantitative assessment

- thyroid gland — in the area comprising the maximum number of counts per pixel;

- analyzed parathyroid gland;

- mediastinal background.

Comparison of the number of counts of the designated ROI allows of following index specification:

- $\mathrm{I}_{\mathrm{T} / \mathrm{B}}$ - average number of counts per pixel in the thyroid gland in relation to background;

- $I_{P / B}$ - average number of counts per pixel in parathyroid in relation to background;

- $\mathrm{I}_{\mathrm{P} / \mathrm{T}}$ - average number of counts per pixel in parathyroid in relation to background.

Comparison of the relationship of counts in early and late images allows the assessment of tracer retention: the absence or presence of a prolonged retention in the parathyroid gland in relation to background and to thyroid gland and in thyroid gland in relation to background. The results are presented in the form of a table (Figure 8).

According to the literature, in case of subtraction examination with use of $\mathrm{Na}^{99 \mathrm{~m}} \mathrm{TcO}_{4} /{ }^{99 m} \mathrm{Tc}-\mathrm{MIBI}$ careful image acquisition is required. It is most seriously limited by motion artifacts. Patient's movement during the examination makes it impossible to analyze the results properly. An example of the effect of inaccurate images adjustment is shown below (Figures 9 and 10).

This issue is particularly important because analyzed images are recorded separately. Motion artifacts are difficult to avoid, because time of patient immobilization during the examination is 35 min. Authors' own data analysis shows that $70 \%$ of the examinations require initial correction.

Commercially available packages for parathyroid scintigraphy analysis do not allow post-correction of these artifacts, which precludes optimal images matching and creates risk of false positive results. Our program allows motion artefacts correction with accuracy of 1 pixel of an image, which often constitutes a positive or negative result of the subtraction scintigraphy.

The program offers a uniform procedure for analyzing parathyroid examinations in diagnostic centers, accelerates the analysis of such examinations performed with use of a single radioactive tracer that require the acquisition of consecutive images of the

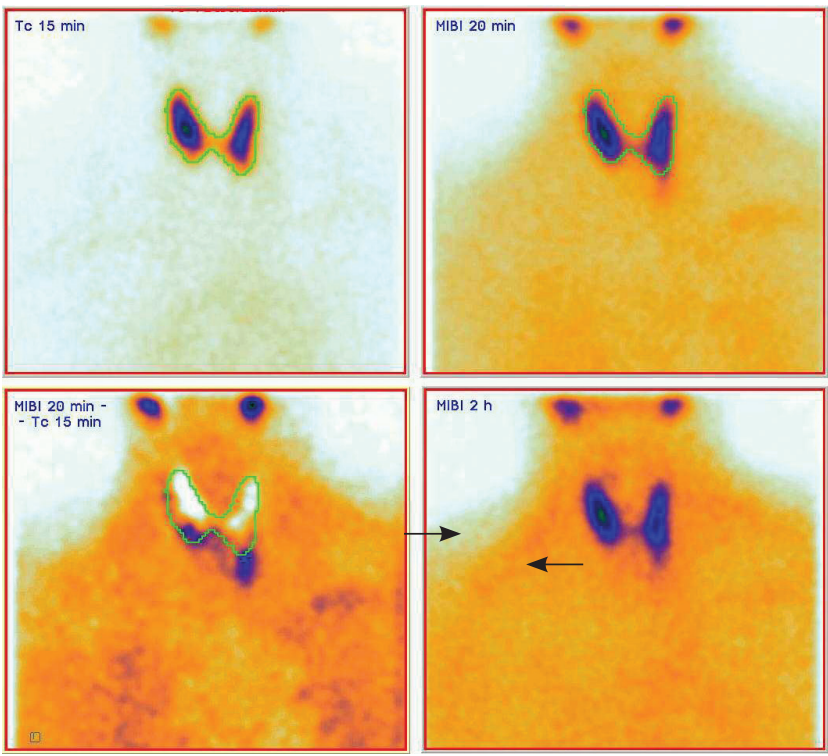

Figure 8. The example of combined $\mathrm{Na}^{99 \mathrm{~m}} \mathrm{TcO}_{4} /{ }^{99 m} \mathrm{Tc}-\mathrm{MIBI}$ subtraction and ${ }^{99 \mathrm{~m} T c-M I B I}$ dual phase scintigraphy: Two foci of tracer accumulation (arrows) matching hypertrophic patathyroids visible only on subtraction scan


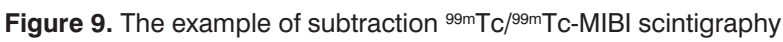
without the motion correction: Single focus of tracer accumulation in lower part of right thyroid lobe observed on subtraction scan is an artefact. The scan result is positive

patient, without altering his body position between successive stages of registration. The program allows the automatization of time-consuming procedures, which saves time and reduces the risk of operator error.

\section{Summary}

There is no controversy in the published literature that sensitivity and specificity of parathyroid scintigraphy is superior to 

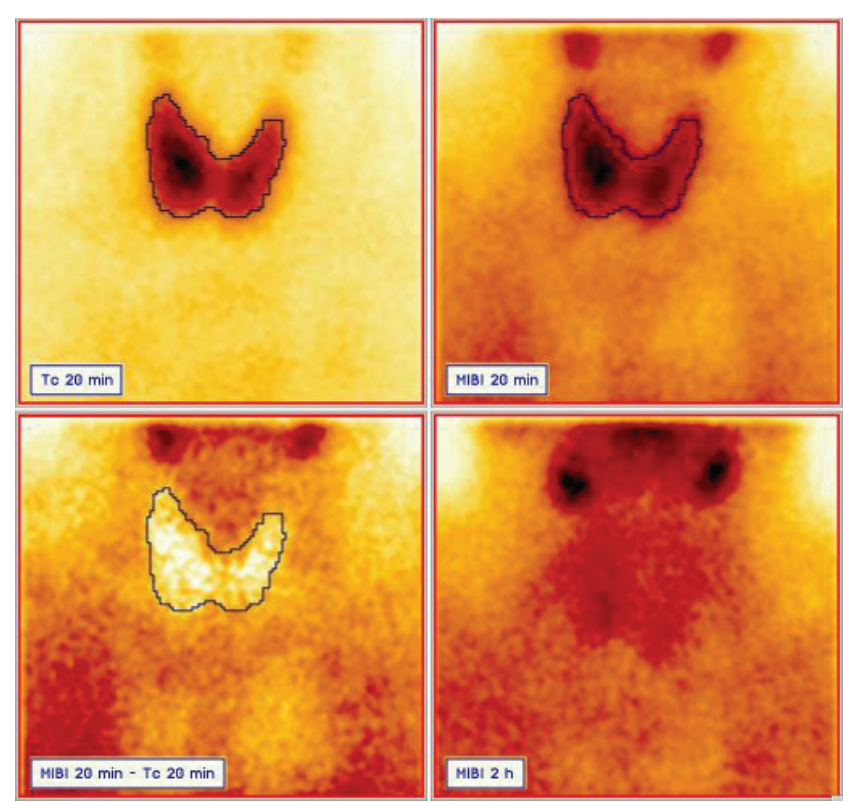

Figure 10. The same example of subtraction $99 \mathrm{mTc} / 99 \mathrm{mTC}-\mathrm{MIBI}$ scintigraphy with the motion correction: No focus of tracer accumulation is visible anymore. The scan result is negative

other imaging techniques. However no uniform protocol has been established for scintigraphy. In order to analyze parathyroid scintigraphic images in the Department of Nuclear Medicine at the Medical University of Warsaw, we have developed a program that allows qualitative and quantitative evaluation of recorded images and motion artifacts correction. This program offers a uniform procedure of analysis of parathyroid imaging results in diagnostic centers, accelerates the analysis of parathyroid tests performed with use of single radioactive tracer, that require the acquisition of consecutive images of the patient, without altering his body position between successive stages of registration. This program allows for automation of previously time consuming procedures and thus saves time and decreases a risk of operator's errors.

\section{References}

1. Peeler BB, MartinWH, Sandler MP, Goldstein RE. Sestamibi parathyroid scaning and preoperative localization studies for patients with recurent/persistent hyperparathyroidism or significant comorbid conditions: development of an optimal localization strategy. Am Surg 1997; 63: 37-46.

2. Bergerfelz A, Tennvall J, Valdermarsson S, Lindblom P, Tibblin S. Sestamibi versus thalium subtraction scintigraphy in parathyroid localization: a prospective comparative study in patients with predominantly mild primary hyperparathyroidism. Surgery 1997; 121: 601-605.

3. Hindié E, Mellière D, Jeanguillaume C, Perlemuter L, Cheehade F, Galle P. Parathyroid imaging using simultaneous double-window recording of technetium-99m-sestamibi and iodine-123. J Nucl Med 1998; 39: 1100-1105.

4. Taillefer R. ${ }^{99 m} T c$ sestamibi parathyroid scintigraphy. In: Freeman LM (Ed.) Nuclear Medicine Annual. Raven Press, New York 1995; 51-79.

5. Mazzeo S, Caramella D, Lencioni R et al. Comparison among sonography, double-tracer subtraction scintigraphy and double phase scintigraphy in the detection of parathyroid lesions. AJR 1996; 166: 1465-1470. 